\title{
A Design of Sigma-Delta ADC Using OTA
}

\author{
Miss. Niveditha Yadav $\mathrm{M}^{1}, \mathrm{Mr}$. Yaseen $\mathrm{Basha}^{2}$, Dr. Venkatesh kumar $\mathrm{H}^{3}$ \\ ${ }^{I}$ Department of ECE, PG Student, NCET/VTU, and Bengaluru, India \\ ${ }^{2}$ Department of ECE, Assistant professor, NCET/VTU, India, Bengaluru \\ ${ }^{3}$ Department of ECE, Associate professor, NCET/VTU, India, Bengaluru
}

\begin{abstract}
Sigma-Delta Analog-to-Digital converter (ADC), is widely used in portable electronic products. An operational transconductance amplifier (OTA) is one of the most important components of ADC. This paper presents a new design of two stages OTA. The design incorporates Sleep insertion technique and leakage feedback current approach for improving design parameters such as gain, and power as compared to earlier work. The design is simulated in $0.18 \mu \mathrm{m}$ CMOS technology with supply voltage $1.8 \mathrm{~V}$.
\end{abstract}

Keywords: ADC, OTA, Sleep insertion Technique, Leakage feedback approach.

\section{INTRODUCTION}

Modern VLSI devices demands for excessive information value with low energy consumption and needless speed. The key additives in the wireless receiver is the ADC, it is far way a margin in the middle of analog and digital design.

\section{Operational Transconductance Amplifier}

The OTA is a basic building blocks found in many analog devices such as data converter's (ADC \&DAC). The OTA is a Transconductance device in which the input voltage controls the output current, it means that OTA is a voltage controlled current source whereas the op-amps are voltage controlled voltage source. An OTA is basically an opamp without output buffer, so it can only drive loads.

\section{Analog-to-digital converter}

$\mathrm{ADC}$ is a fundamental block in mixedsignal VLSI circuits. The rapid growth of mobile electronic systems increases the demand for developing low-cost and low-power circuit technique with high performance. Sigma delta $(\Sigma \Delta)$ modulators are one of the preferred architectures for high resolution converters. Power consumption and area are the key parameters for a sigma delta modulator these parameters cannot be changed once an ADC is designed. While it can operate at higher speed and will consume less power when operating at a lower resolution.

\section{PROPOSED OTA ARCHITECTURE}

OTA is one of the basic building blocks of any analog circuit. OTA is in existence since very long time, this is not a recent technology. An OTA has all the characteristics of an operational voltage amplifier except that the output impedance ideally approaches infinity rather than zero. OTA is used to form the R-C integrator, which is the key block of $\Sigma \Delta$ modulator. An Two-Stage OTA topology with rail to-rail output swing is adopted for low voltage, low power designs. Two stage OTA is a configuration two stages are used. One of them provides high gain followed by second stage which provides high voltage swing. This modification increases the gain compared to single stage OTA. But increases complexity of design, Hence reduce the speed as compared to single stage amplifier[2].

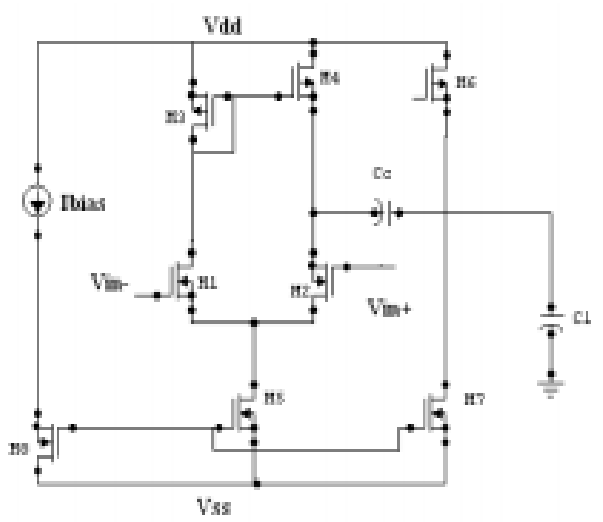

Fig 1: Two stage OTA

\section{BLOCK DIAGRAM OF ADC ARCHITECTURE}

Fig 2. Shows The block diagram of a first order $\Sigma \Delta$ modulator which consists of a integrator, a comparator, which acts as an ADC and 1-bit DAC, which is placed in the feedback loop. The name first order is derived from the information that there is only one integrator in the circuit, placed in the forward path. When the output of the integrator is positive, the comparator feeds back a positive reference signal that is subtracted from the input 
signal of the integrator. Similarly, when the integrator output is negative, the comparator feeds back a negative signal that is added to the incoming signal

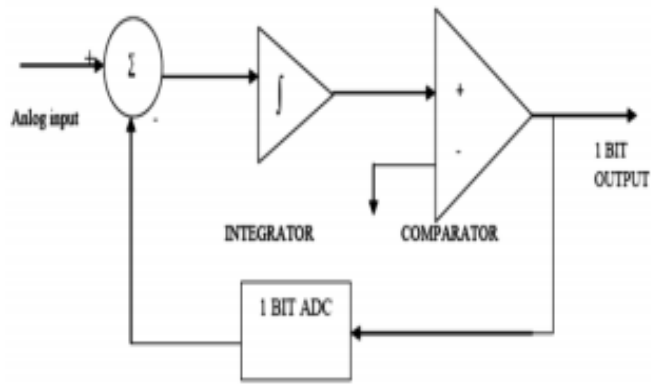

Fig 2. 1 bit Sigma-delta Modulator.

\section{METHODOLOGY}

sleep insertion technique and Leakage feedback approach is to reduce tha power consumption, leakage current and to increase the circuit performance.

\section{1) Sleep Insertion Technique}

Sleep approach is used to rail off the circuit from Vdd to ground, so insert a PMOS transistor above pull up network and Vdd and NMOS transistor below pull down Network and GND. During standby mode a sleep transistor turns off turn off and rail from Vdd and reduces the leakage current. During active mode $\mathrm{ON}$ the sleep transistor and direct connection of circuit with Vdd, so increase the performance of the circuit and Reduces the leakage power.

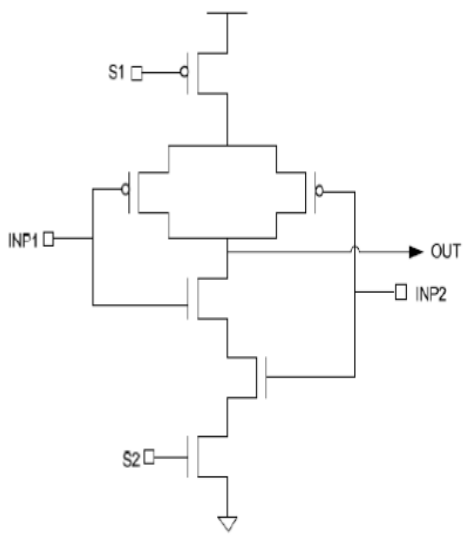

Fig4. sleep insertion technique

\section{2) Leakage Feedback Approach}

Leakage reduction technique is leakage feedback approach; In this approach two parallel PMOS transistor above pull up network and Vdd. To provide the inverting output of the circuit connects a inverter at the output, an inverter provides the proper logic feedback to both pull down $\operatorname{NMOS}\left(\mathrm{S}^{\prime}\right)$ and pull up PMOS(S) sleep transistor. This two transistor enhance the circuit performance and maintain the proper logic of the circuit during standby mode.

In standby mode one of the transistor of parallel sleep transistor turn off both NMOS and PMOS, the output of the circuit is pass through inverter which keep $\mathrm{ON}$ one of the sleep transistor which is connected parallel by providing the proper feedback approach. Hence circuit is active in standby mode, the various leakage current which flow during standby mode and increase the performance of the circuit

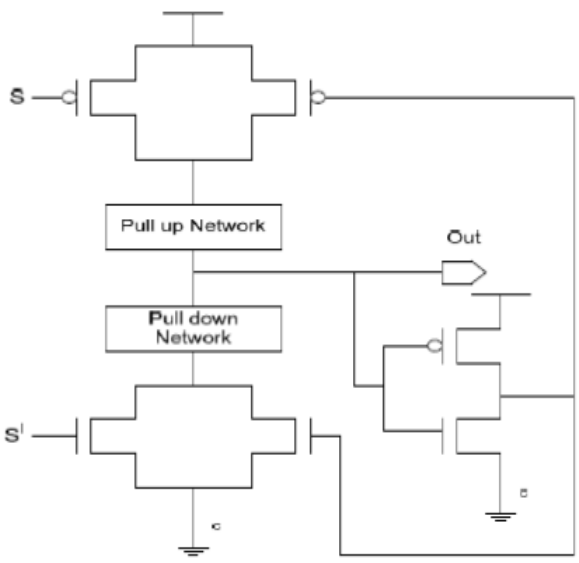

Fig.5: leakage feedback current approach

\section{V.SIMULATION RESULT AND DISSCUSSION 1) DESIGN OF COMPARATOR}

Comparator is one of the fundamental building blocks in most analog to digital converters (ADCs). High speed flash ADCs, require high speed, low power and small chip area. Comparators are known as 1-bit analog to digital converter and hence they are mostly used in large abundance in $A / D$ converter [4]. A comparator is same as that like of an operational amplifier in which they have two inputs (inverting and non-inverting) and an output. The function of a CMOS comparator is to compare an input signal with a reference signal which produces a binary output signal [4].

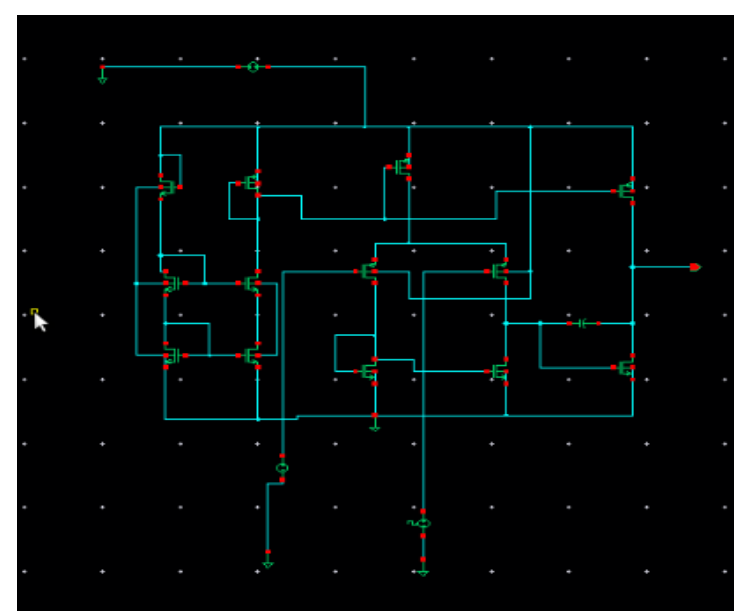

Fig.6: Schematic view of comparator 


\section{Simulation Results}

From the analysis, the Gain of the design is $3.8 \mathrm{~dB}$ and the static and total power consumption of design is $155.5 \mu \mathrm{W}$ and $103.1 \mu \mathrm{W}$.

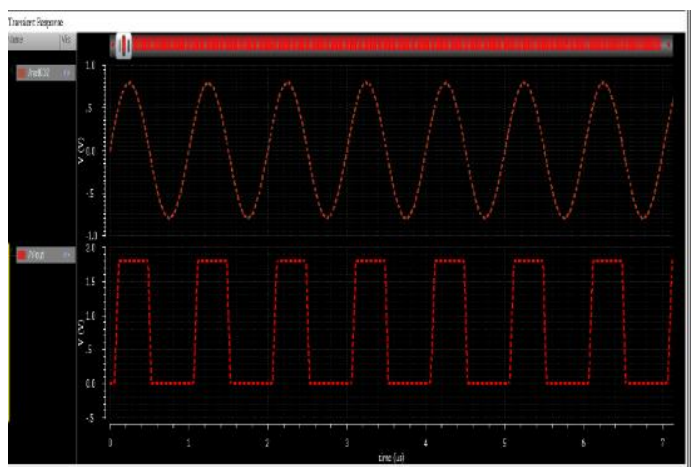

Fig.7: Result of comparator

\section{2) DESIGN OF 1-BIT DAC}

This circuit contains two transmission gates and an inverter, two reference voltages. For particular case, + Vref is taken as $+1.8 \mathrm{~V}$ and - Vref is taken as $-1.8 \mathrm{~V}$. And the operation of the circuit can be explained by two cases.

If the input is 1 , then output of the DAC is + Vref and if the input is 0 , then DAC output is Vref. This logic is implemented using a $2 \times 1$ multiplexer circuit. Output of the comparator act as the select lines of the multiplexer to select the 1-bit digital input. Transmission gates are controlled by the output of comparator and its inverted output is obtained from the inverter [1]

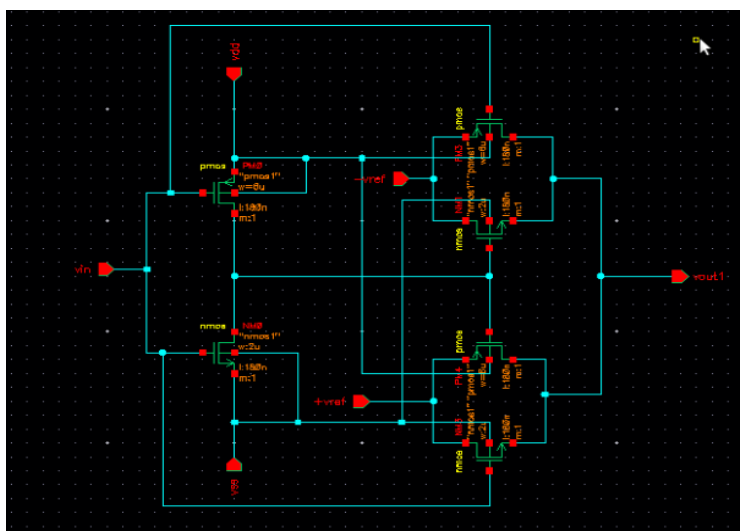

Fig.8: Schematic view of 1-bitDAC

\section{Simulation Results}

From the analysis, the Gain of the design is $7.75 \mathrm{~dB}$ and the static and total power consumption of design is $2.343 \mu \mathrm{W}$ and $2.726 \mu \mathrm{W}$.

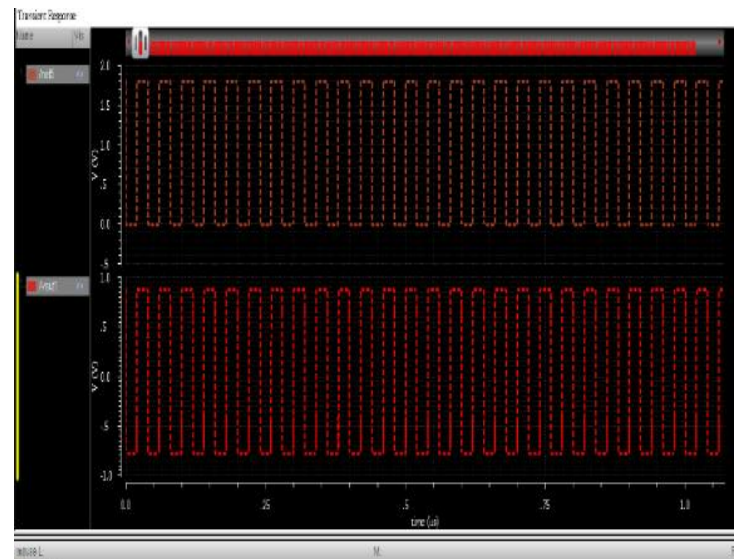

Fig.9: Result of 1-bit DAC

\section{3) DESIGN OF TWO STAGE OTA}

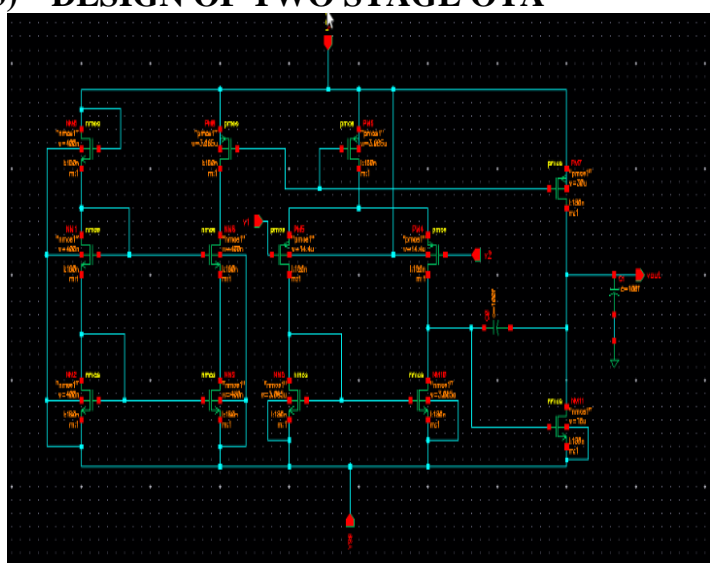

Fig.10: Schematic view of Two-Stage OTA

\section{Simulation Results}

From the analysis, the Gain of the design is $9.6 \mathrm{~dB}$ and the static and total power consumption of design is $15.16 \mathrm{~mW}$.

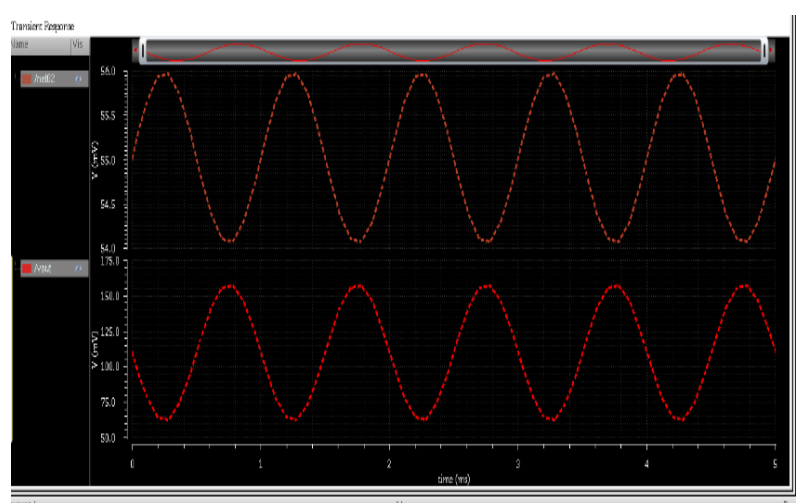

Fig.11: Result of Two-Stage OTA

\section{4) DESIGN OF OP-AMP}

An operational amplifier (often op-amp) is a DCcoupled high-gain electronic voltage amplifier with a differential input and, usually, a single-ended output[11] 


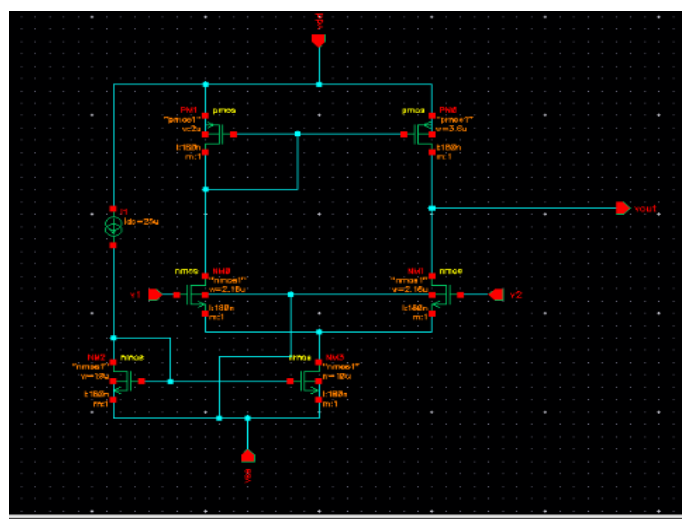

Fig 12 : Schematic view of Op-amp

\section{Simulation Results}

From the analysis, the Gain of the design is $2.9 \mathrm{~dB}$ and the static and total power consumption of design is $118 \mu \mathrm{W}$ and $118.06 \mu \mathrm{W}$.

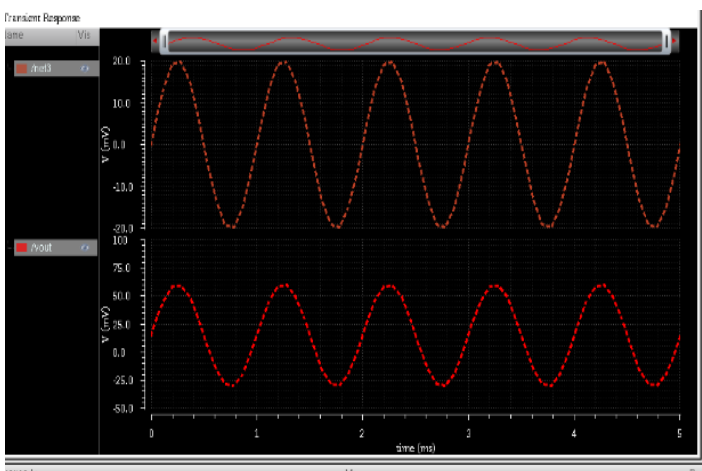

Fig.13: Result of Op-amp

\section{5) OP-AMP INTEGRATOR CIRCUIT}

By replacing this feedback resistance with a capacitor we now have an R-C Network connected across the operational amplifiers feedback path producing another type of operational amplifier circuit commonly called an Op-amp integrator circuit as shown in below figure[11].

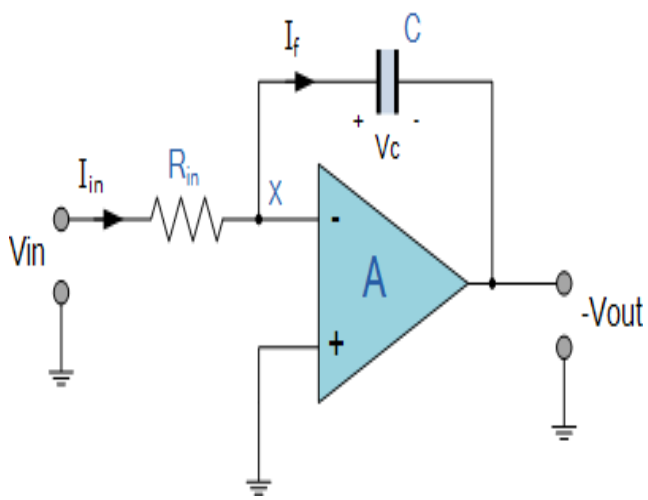

Fig.14: Op-Amp

Op-amp Integrator is an operational amplifier circuit that performs the mathematical operation of Integration, that is we can cause the output to respond to changes in the input voltage over time as the op-amp integrator produces an output voltage which is proportional to the integral of the input voltage.

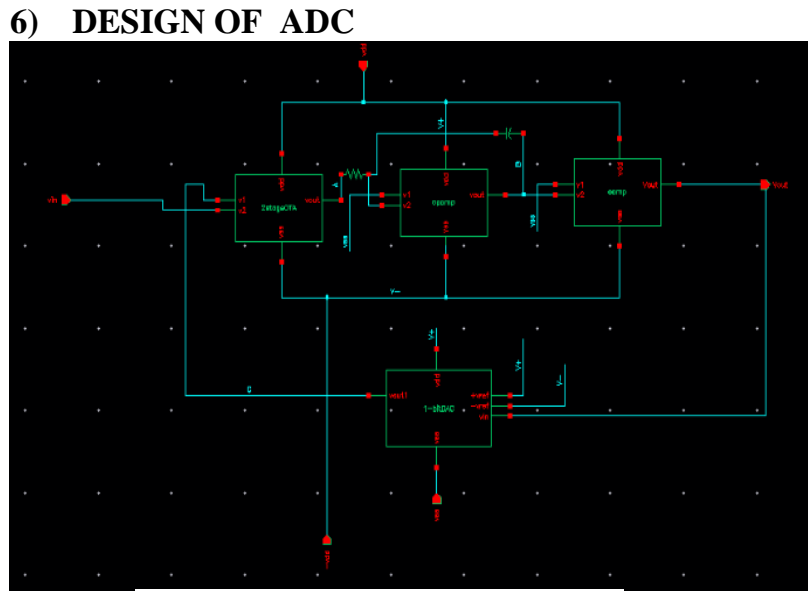

Fig 15 :Schematic of view of ADC

\section{Simulation Results}

From the analysis, the Gain of the design is $6.9 \mathrm{~dB}$ and the static and total power consumption of this ADC design $19.14 \mathrm{~mW}$ and $19.87 \mathrm{~mW}$.

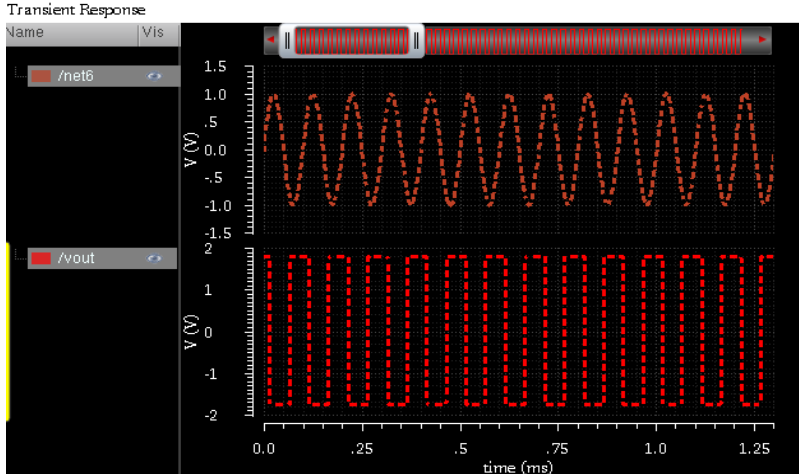

Fig 16: Results of ADC

7) DESIGN OF ADC BY SLEEP INSERTION TECHNIQUE

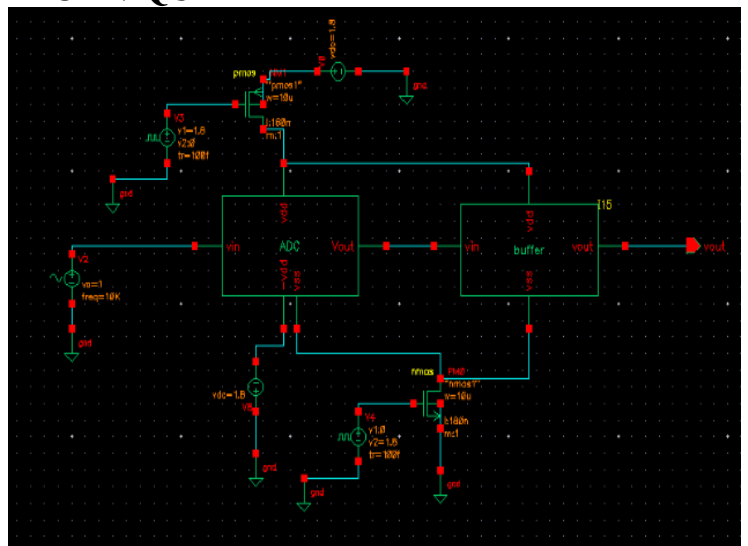

Fig 17: Schematic View of ADC by sleep Insertion Technique 


\section{Simulation Results}

From the analysis, the Gain of the design is $6.9 \mathrm{~dB}$, and static and Total power consumed by OTA is $655.4 \mu \mathrm{W}$.

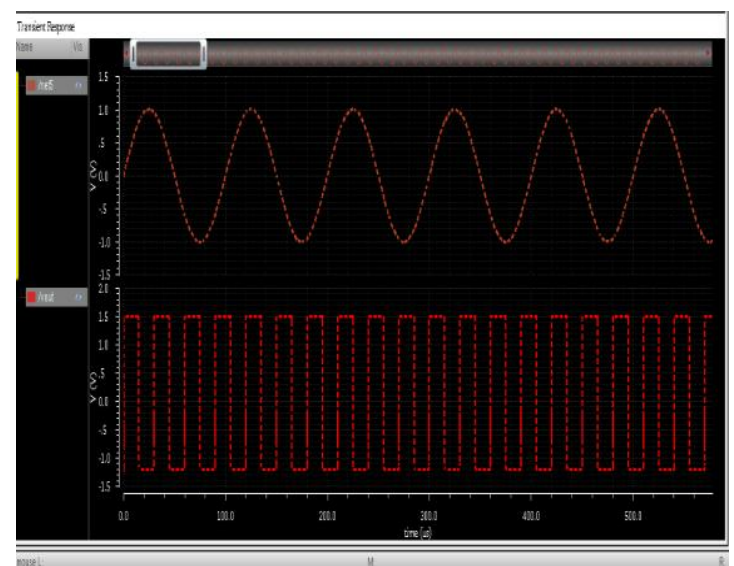

Fig 18: Result of ADC by sleep Insertion Technique

\section{8) DESIGN OF ADC BY LEAKAGE FEEDBACK APPROACH}

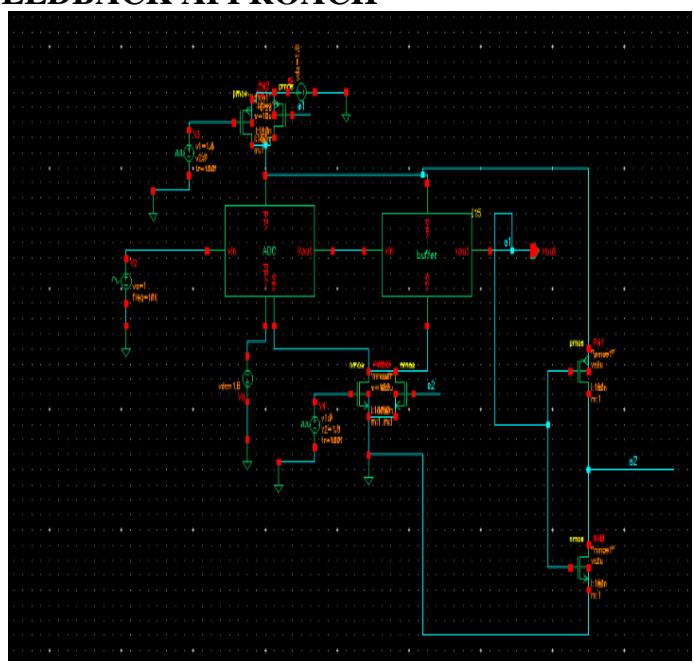

Fig19: Schematic view of ADC by Leakage Feedback Approach

\section{Simulation Results}

From the analysis, the Gain of the design is $6.9 \mathrm{~dB}$, and static and total power consumed by OTA is $11.38 \mathrm{~mW}$

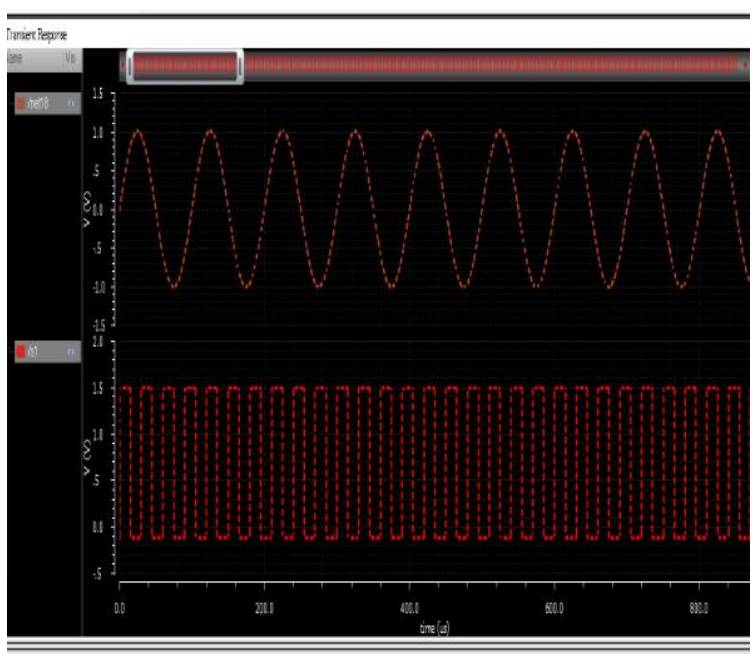

Fig 20: Schematic view of ADC by Leakage Feedback Approach

\section{COMPARITION TABLE}

\begin{tabular}{|l|l|l|l|}
\hline DESIGN & $\begin{array}{l}\text { STATIC } \\
\text { POWER } \\
(\mathrm{W})\end{array}$ & $\begin{array}{l}\text { TOTAL } \\
\text { POWER } \\
(\mathrm{W})\end{array}$ & GAIN \\
\hline TWO-STAGE OTA & $15.16 \mathrm{~m}$ & $15.16 \mathrm{~m}$ & $9.6 \mathrm{~dB}$ \\
\hline OP-AMP & $118 \mu$ & $118.06 \mu$ & $2.9 \mathrm{~dB}$ \\
\hline 1-BIT DAC & $2.343 \mu$ & $2.726 \mu$ & $7.75 \mathrm{~dB}$ \\
\hline COMPARATOR & $155.5 \mu$ & $103.1 \mu$ & $3.8 \mathrm{~dB}$ \\
\hline ADC & $19.14 \mathrm{~m}$ & $19.87 \mathrm{~m}$ & $6.9 \mathrm{~dB}$ \\
\hline $\begin{array}{l}\text { ADC BY SLEEP } \\
\text { INSERTION } \\
\text { TECHNIQUE }\end{array}$ & $655.4 \mu$ & $655.4 \mu$ & $6.9 \mathrm{~dB}$ \\
\hline $\begin{array}{l}\text { ADC BY LEAKAGE } \\
\text { FEEBBACCURRENT }\end{array}$ & $11.38 \mathrm{~m}$ & $11.38 \mathrm{~m}$ & $6.9 \mathrm{~dB}$ \\
\hline
\end{tabular}

\section{CONCLUSION}

In this work, two- stage OTA is designed using a sleep insertion technique and leakage feedback approach. The design is carried out in $0.18 \mu \mathrm{m}$ CMOS technology with supply voltage is $1.8 \mathrm{~V}$. The obtained Gain of the design with sleep insertion technique is $6.9 \mathrm{~dB}$, and total power consumed is $655.4 \mu \mathrm{W}$. The obtained Gain of the design with leakage feedback current approach is $6.9 \mathrm{~dB}$, total power consumed is $11.38 \mathrm{~mW}$. The designed OTA is incorporated in Sigma-Delta $\mathrm{ADCs}$ for better performance.

\section{REFERENCE}

[1] "Design of 1-Bit for Delta-Sigma Modulator", Remya Thankachan PG Scholar Dept.of ECE College of Engineering, Munnar Jayakrishnan K.R Assistant Professor Dept.of ECE College of Engineering Munnar ,Shahana T.K, PhD Associate Professor Div.of Electronics SoE, CUSAT -International Conference on Emerging Trends in Technology and Applied Sciences (ICETTAS 2015). 
[2] "Performance Evaluation of Different Type of CMOS Operational Transconductance Amplifier", International Journal of Science and Research (IJSR), India Online ISSN: 2319-7064.

[3] [3] "Design, Simulation and Power Analysis of Sigma-Delta Modulator using $0.18 \mu \mathrm{m}$ CMOS Technology", Archana Parutabadia*, Channakka L.a aDepartment of Electronics \& Communication Engineering SDMCET Dharwad,India International Journal of Current Engineering and Technology.

[4] "Design and Performance Analysis of a Double-Tail Comparator for Low-Power Applications",IJSRET NOV-2014

[5] KushGulati and Hae-Seung Lee, "A HighSwing, High-Performance CMOS Telescopic Operational Amplifier", IEEE Journal of Solid-State Circuits, Vol. 33, No. 12, December 1998.

[6] R.Jacob Baker, Harry W. Li \& David E. Boyce, "CMOScircuit design, layout and simulation", IEEE Press Serieson Microelectronic Systems, Prentice Hall of IndiaPrivate Limited, 2004.

[7] Zahra Haddad Derafshi and Mohammad HosseinZarifi, "Low-Power High-Speed OTA in $0.35 \mu m$ CMOS Process", European Journal of Scientific Research, ISSN 1450216X Vol.37 No.3 (2009), pp.368-375

[8] D.Nageshwarrao, S.VenkataChalamand, V.MalleswaraRao, "Gain Boosted Telescopic OTA with 110db Gain And 1.8GHz. UGF", International Journal of Electronic Engineering Research, ISSN 0975 - 6450 Volume 2 Number 2 (2010) pp. $159-166$

[9] CarstenWulff, TrondYtterdal, "High Speed, High Gain OTA in a Digital 90nmCMOS Technology", Department of Electronics and Telecommunication, Norwegian University of Science and Technology, N7491 Trondheim, Norway

[10] "Reduction of Leakage Power in CMOS circuits (Gates) using Variable Body Biasing with sleep insertion Technique",1. Sunita Yadav, M.Tech. Student of VLSI Design Department, UTU Dehradun, UK India, 2.Vishal Ramola, Assist. Prof. VLSI Design Departmen[

[11] "Operational Amplifiers (Op Amps)", Professor Katherine Candler Notes courtesy of Professor Sarah Harris.t, UTU, Dehradun, UK India2 